\title{
MANAGING AND MITIGATING HOUSING - PRICE PREDICTIONS USING A GENETIC ALGORITHM: A CASE OF KING COUNTY IN WASHINGTON, U.S.A
}

\author{
Uma Gunasilan \\ Associate Professor, Hult International Business School, London, United Kingdom.
}

\begin{abstract}
The current research aims to establish the effects of using machine learning algorithms to optimize predictions of housing prices. For the purpose of the study, dataset from the King County, Washington, United States of America is used. The conclusions the research expects to draw are to find a optimized algorithm to measure the success of housing price prediction during an increasingly hyper turbulent environment that are increasingly becoming more common. The approach is by applying a comparison mechanism between features selection and feature extraction in the predictions.
\end{abstract}

Key words: Principal component analysis (PCA), The Akaike information criterion (AIC), Bayesian Information Criterion (BIC), Machine Learning prediction, Real Estate Price Prediction

Cite this Article: Uma Gunasilan, Managing and Mitigating Housing - Price Predictions using a Genetic Algorithm: A Case of King County in Washington, U.S.A, International Journal of Management (IJM), 12(9), 2021, pp. 9-18.

https://iaeme.com/Home/issue/IJM?Volume=12\&Issue=9

\section{INTRODUCTION}

Owning a house is one of the biggest dreams of the majority of people and one of the largest, most expensive purchases in a person's life. However, it is often difficult to determine or predict housing prices. This project aims to build a prediction model to predict housing prices, and it can help homebuyers and home sellers to make the best decision. This project used the housing sales dataset in King County, USA, which includes 21,613 entities with 20 explanatory features 
Managing and Mitigating Housing - Price Predictions using a Genetic Algorithm: A Case of King County in Washington, U.S.A

\section{LITERATURE REVIEW}

\subsection{Nature of Work}

Due to the analysis in the literature review, this project addressing some weaknesses in previse work in using $\mathrm{KC}$ housing price dataset that found with $\mathrm{Wu}[8]$ and Cai[9], By redesign those methodologies. This project using different algorithms and evaluation matrices. I addition, adding some change and improvement by using information criteria approaches Akaike information criterion (AIC) and Bayesian information criterion (BIC) as a type of feature selection. which is not used in the $\mathrm{Wu}[8]$ and Cai[9] studies. After that, we make a comparison between the feature returned by using feature extraction PCA [8], and feature selection returned by AIC and BIC. The algorithms that chosen to build a prediction model are SVR [8], RF, GB and linear regression [9]. In the evaluation of the model performance, we use 4 types of evaluation matrices MAE, MSE, RMSE and R - square. After that, I will use parameter optimizing with the best algorithms that obtained the lowest error rate and highest score in evaluation matrices by using GA[5], which is not used in the $\mathrm{Wu}[8]$ and Cai[9] studies.

In the recent papers that forecasting real estate prices based on using machine learning algorithms. These algorithms generating models have been used in different aspects, estimating an appropriate price level for a new real estate, forecasting properties sale price [1],[2]. There are many research studies used regression model, were regression get the most highly significant experimentation results [3],[5]. They used deferent types of regression modelling, exponential, linear and genetic regression, genetic was the highest accuracy [3]. In improving performance of Regression the spatio-temporal analysis techniques used to increase Regression performance [4].

In using SVM algorithm,[8]suggested different models predict real estate price based on support vector machine (SVM) and particle swarm optimization(PSO).the experiment results shown by PSO-SVM has better predicting performance when they compared with genetic and grid algorithms, on another hand [9] used a support vector machines hybrid with genetic algorithm in the result was predicting housing price, the experiment results have shown SVM$\mathrm{G}$ has predicting accuracy than using traditional methods. The most common methods are artificial neural network and genetic algorithms as they have better self-learning and adaptive capacity [6], [7].

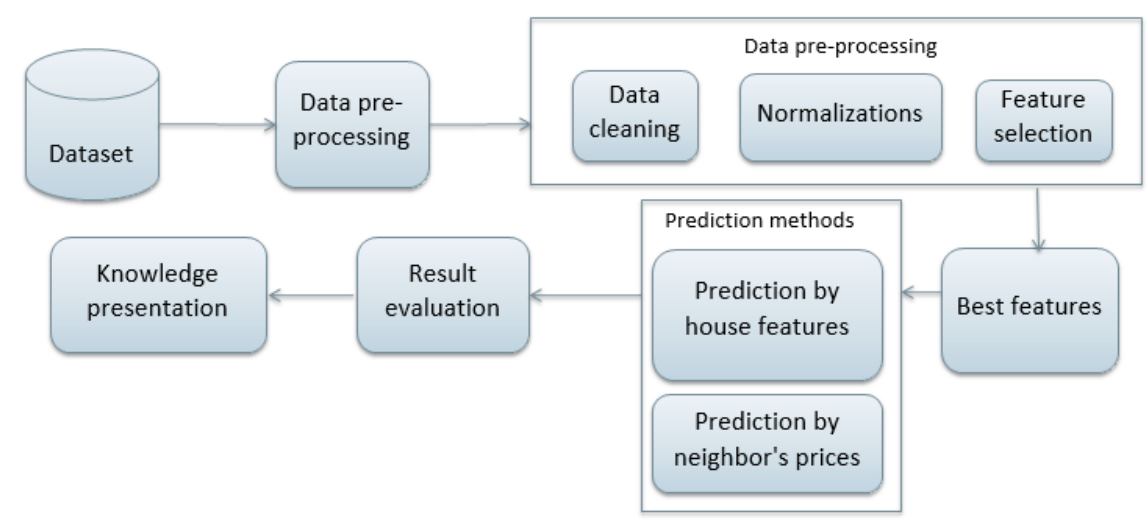

Figure 1 House Price Prediction Methodology

Using feature selection techniques with AIC and BIC will allow the SVR, RF, GB and linear regression algorithms to have at least a 5\% lower RMSE than using the same algorithms with 
feature extraction in PCA. Using feature selection techniques with AIC and BIC will not allow SVR, RF, GB and linear regression algorithms to have at least a 5\% lower RMSE than using the same algorithms with feature extraction in PCA.

Using feature selection techniques with AIC and BIC will allow the SVR, RF, GB and linear regression algorithms to have at least a 5\% higher R-squared score than using the same algorithms with feature extraction in PCA. Using feature selection techniques with AIC and BIC will not allow the SVR, RF, GB and linear regression algorithms to have at least a 5\% higher R-squared score than using the same algorithms with feature extraction in PCA.

\section{METHODOLOGY AND DERIVATION}

The experiment makes comparisons between 2 methods. One is with feature extraction techniques(PCA), The second with feature selection techniques (AIC and BIC).

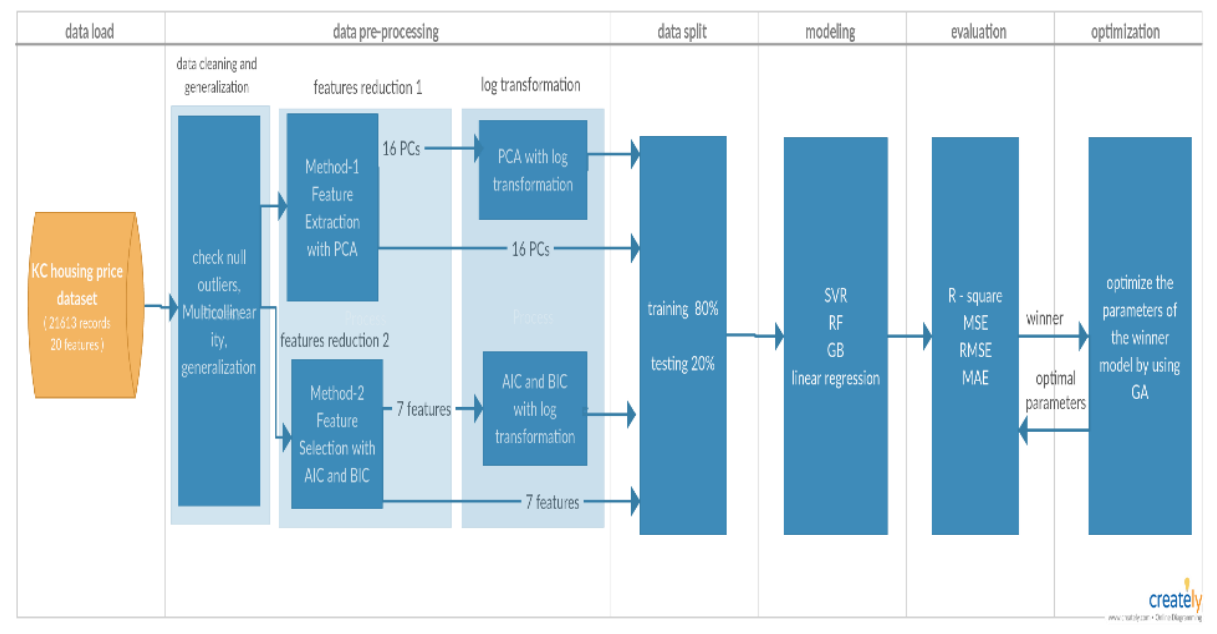

Figure 2 Methodology and Selection Process for Feature Extraction in House Price Prediction

\subsection{Dataset}

The dataset contains housing prices for King County houses sold May 2014- May 2015. This data sets has 21,613 records and 21 variables. The variables describe features of each house, including house price and whether the house was available on the Kaggle website which is website of publish data sets [9]. The table below shows each feature name, description and data type.

Data features considered in the dataset categorically are,

Waterfront view

No waterfront view

Number of bedrooms

Number of bathrooms

Number of floors

View

Condition

Grade of house

price

Sqft living

Sqft lot 
Managing and Mitigating Housing - Price Predictions using a Genetic Algorithm: A Case of King County in Washington, U.S.A

Sqft above

Sqft basement

Yr built

Yr renovated

Zipcode

Lat

Long

Sqft living15

Sqft lot15

\subsection{Data Features Visualization}
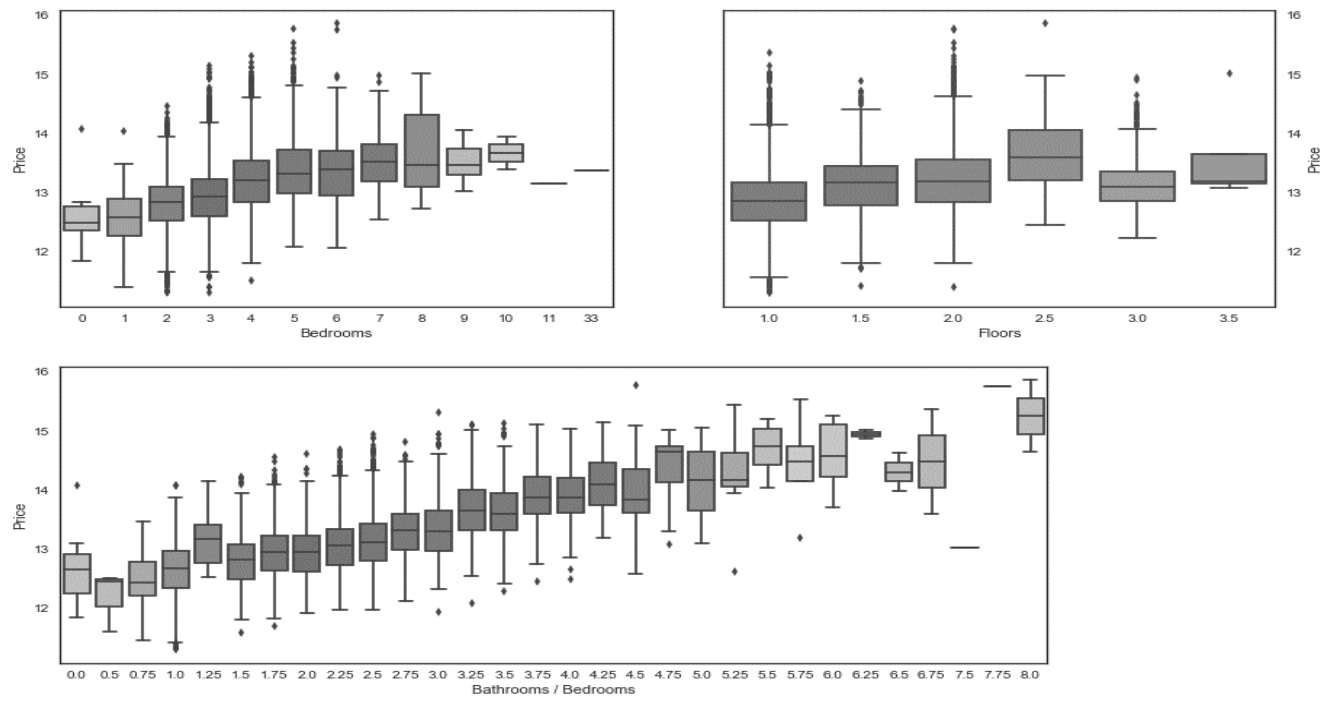

Figure 3 Visualization of Extracted Features

\subsection{Applying Log Transformation}

Let us take price as example to show how we can address the outlier and the high skewness. we can clearly see positive skewing in the distribution of price values in the left figure. The degree of skewing is equal to 4.028 , which is a high number.
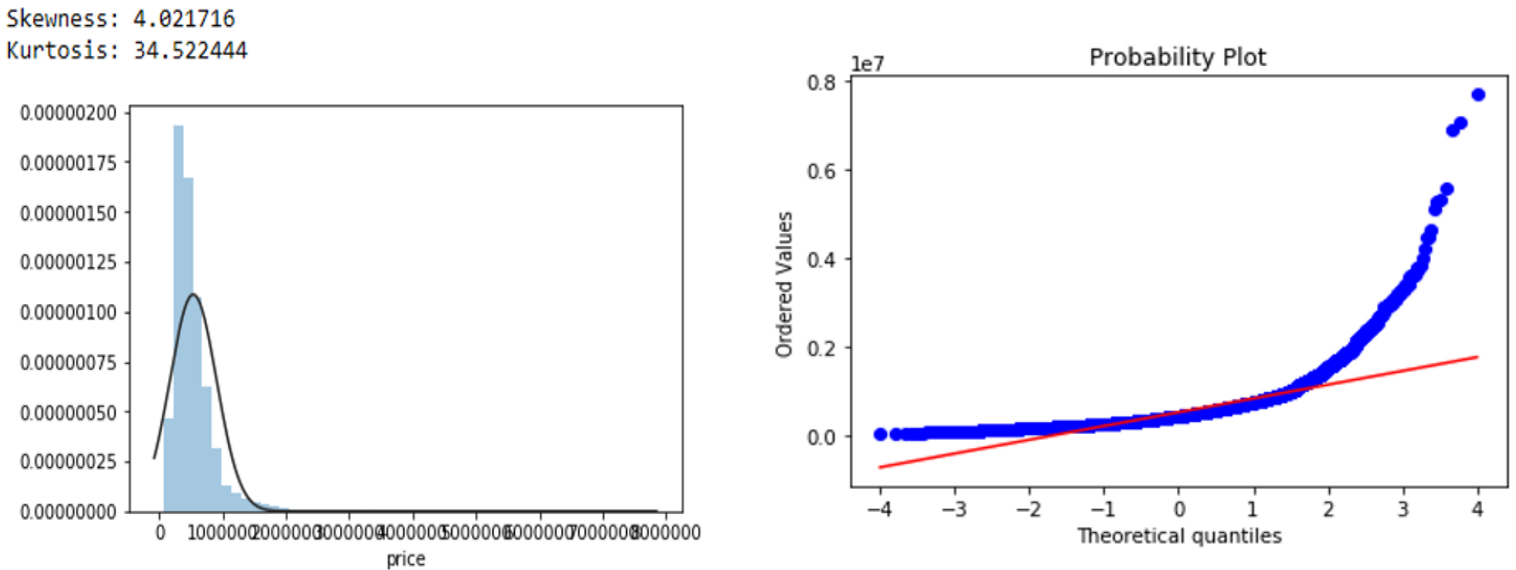

Figure 4 Price Distribution According to Prediction Model Before Log Transformation 
After log transformation, the price distribution becomes more normal, with 0 standard deviation and skewing reduced from 4.02 to 0.428 , also the palpability distribution show more evidence about how price value become more linearly regression. Due to that, we expected that could improve prediction model performance.
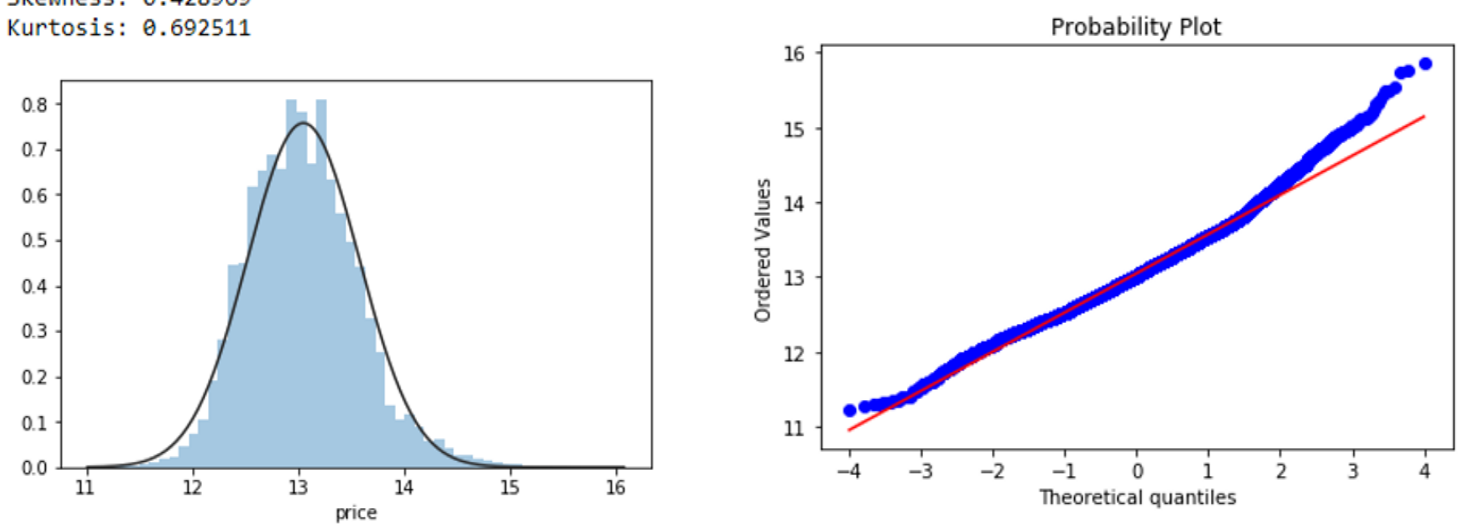

Figure 5 Price Distribution According to Prediction Model After Log Transformation

\subsection{Applying Feature Reduction}

\section{First - PCA (Principal Component Analysis)}

Principal component analysis is a statistical procedure that uses an orthogonal transformation to convert a set of observations of possibly correlated variables into a set of values of linearly uncorrelated variables called principal components.

PCA is convert a set of observations into a set of values of linearly uncorrelated variables called PCs and we calculate the covariance matrix, to get the eigenvalues and eigenvectors as formula below;

$$
\operatorname{var}(X)=\frac{\sum_{i=1}^{n}\left(X_{i}-\bar{X}\right)\left(X_{i}-\bar{X}\right)}{(n-1)}
$$

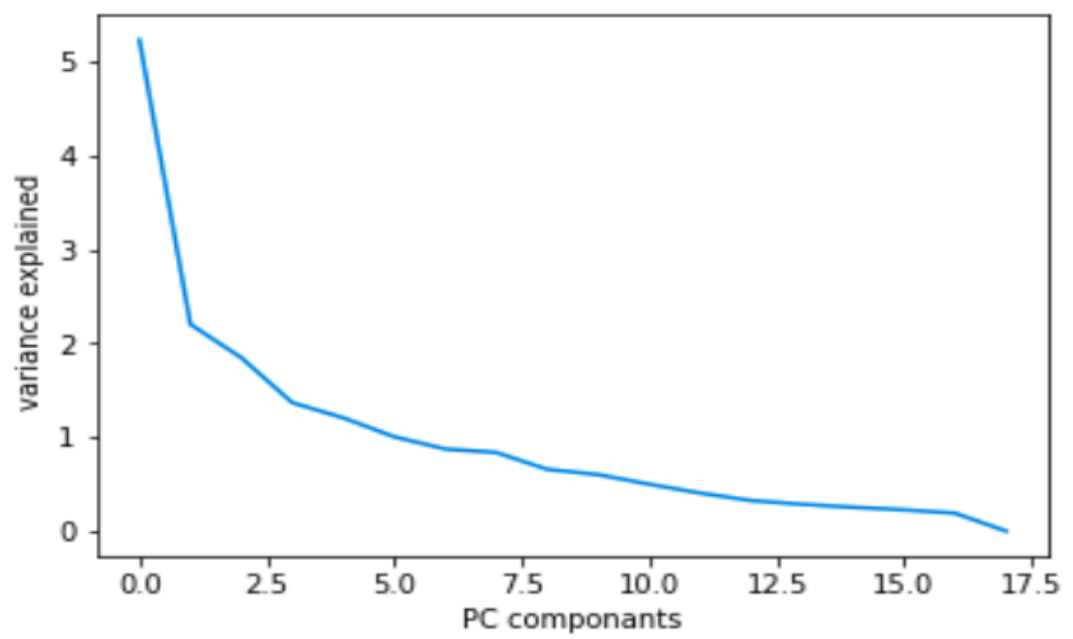

Figure 6 Plots of Each PC's Eigenvalues 


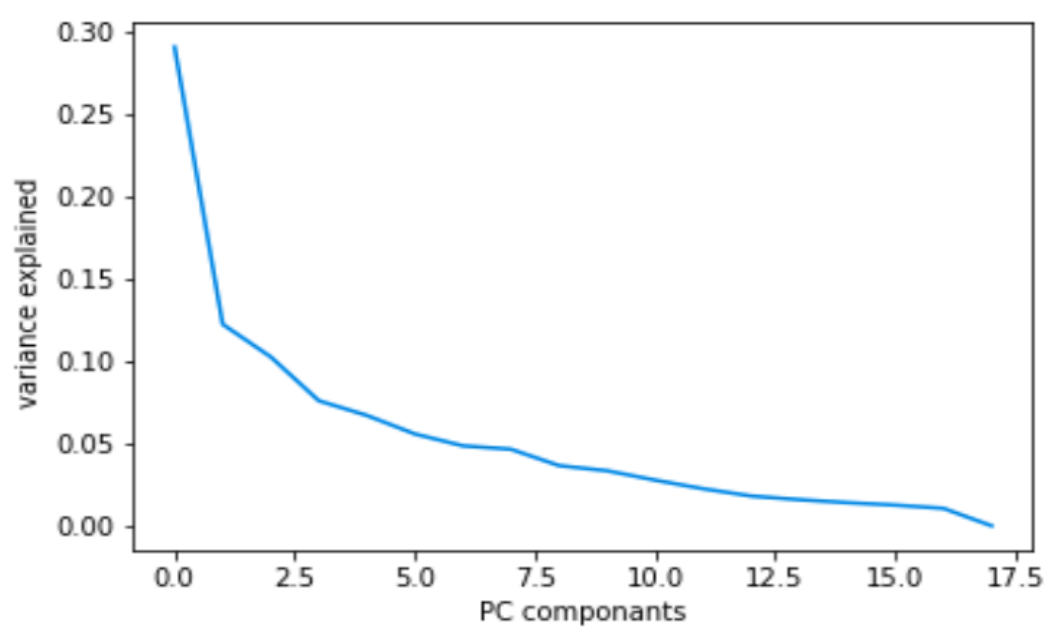

Figure 7 Plots of Variance Ratio Plot

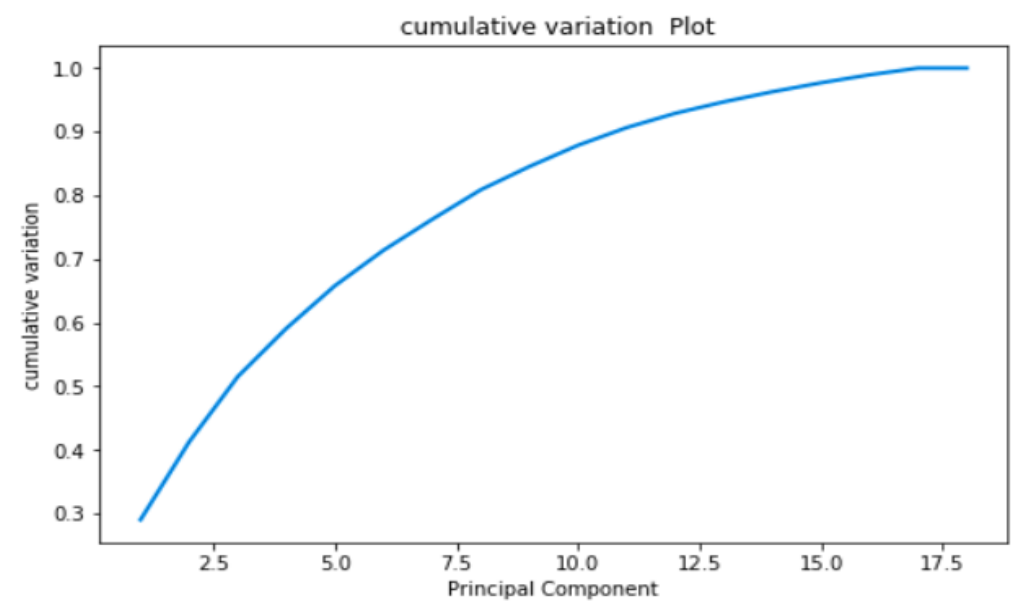

Figure 8 Plots of Explained Variance Cumulative Plot

\section{Second - AIC (Akaike Information Criterion) and BIC (Bayesian Information Criterion)}

The Akaike information criterion (AIC) is an estimator of the relative quality of statistical models for a given set of data. Given a collection of models for the data, AIC estimates the quality of each model, relative to each of the other models. Thus, AIC provides a means for model selection.

AIC is founded on information theory. When a statistical model is used to represent the process that generated the data, the representation will almost never be exact; so some information will be lost by using the model to represent the process. AIC estimates the relative amount of information lost by a given model: the less information a model loses, the higher the quality of that model.

In estimating the amount of information lost by a model, AIC deals with the trade-off between the goodness of fit of the model and the simplicity of the model. In other words, AIC deals with both the risk of overfitting and the risk of underfitting. 
Table 1

\begin{tabular}{|l|c|c|}
\hline \multicolumn{1}{|c|}{ Feature Included } & AIC & BIC \\
\hline grade & $1.89 \mathrm{E}+04$ & $1.89 \mathrm{E}+04$ \\
\hline sqft_living & $2.05 \mathrm{E}+04$ & $2.05 \mathrm{E}+04$ \\
\hline sqft_living15 & $2.32 \mathrm{E}+04$ & $2.32 \mathrm{E}+04$ \\
\hline bathroom & $2.58 \mathrm{E}+04$ & $2.58 \mathrm{E}+04$ \\
\hline lat & $2.88 \mathrm{E}+04$ & $2.88 \mathrm{E}+04$ \\
\hline view & $3.09 \mathrm{E}+04$ & $3.09 \mathrm{E}+04$ \\
\hline bedroom & $3.09 \mathrm{E}+04$ & $3.09 \mathrm{E}+04$ \\
\hline sqft_basement & $3.14 \mathrm{E}+04$ & $3.14 \mathrm{E}+04$ \\
\hline
\end{tabular}

\subsection{Modelling using SVR, RF, GB and Linear Regression}

Supervised learning models with associated learning algorithms that analyze data used for classification and regression analysis. The research will build an ensemble of deep independent trees and it will build an ensemble of shallow and weak successive trees with each tree learning and improving on the previous. The research will also use linear regression as a method to find pattern with a Best Fit Line.

Inspired by nature, specifically evolution (such as natural selection, reproduction, and survival of the fittest). This will be used in this research to optimize algorithm's parameter.

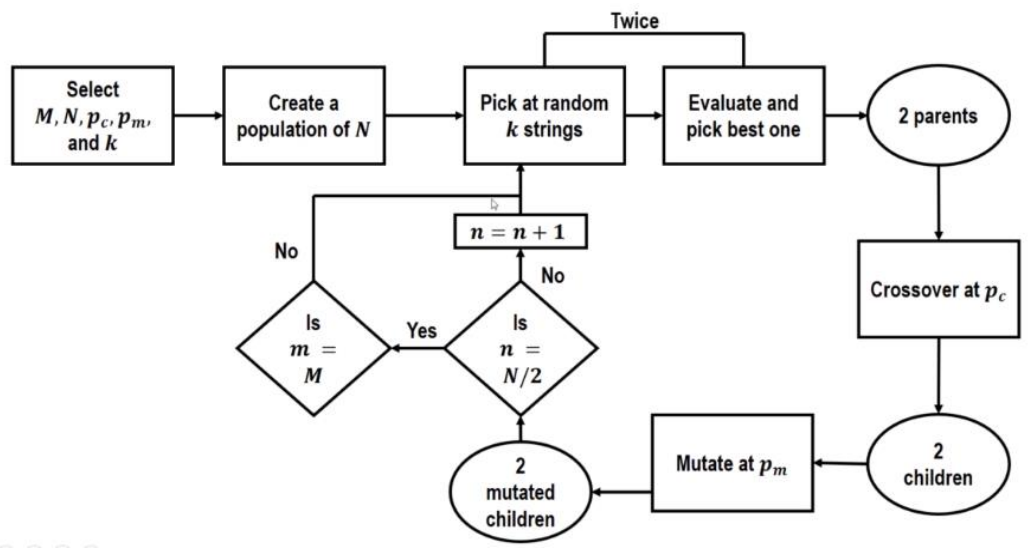

Figure 9 The Methodolgoy Used for the Generic Algorithm

\section{RESULTS AND FINDINGS}

\subsection{PCA Application Results}

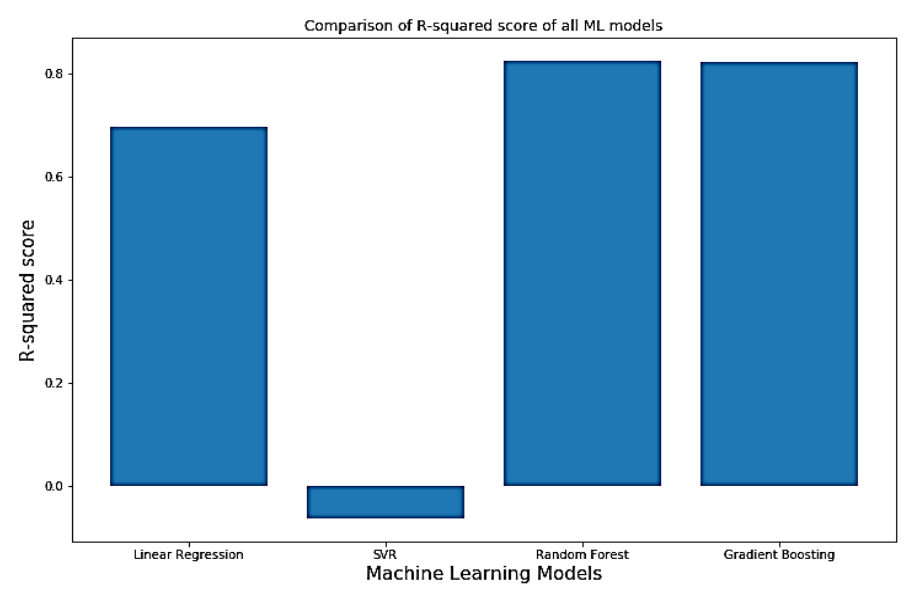

Figure 10 Results of PCA Before Log Transformation 
Managing and Mitigating Housing - Price Predictions using a Genetic Algorithm: A Case of King County in Washington, U.S.A

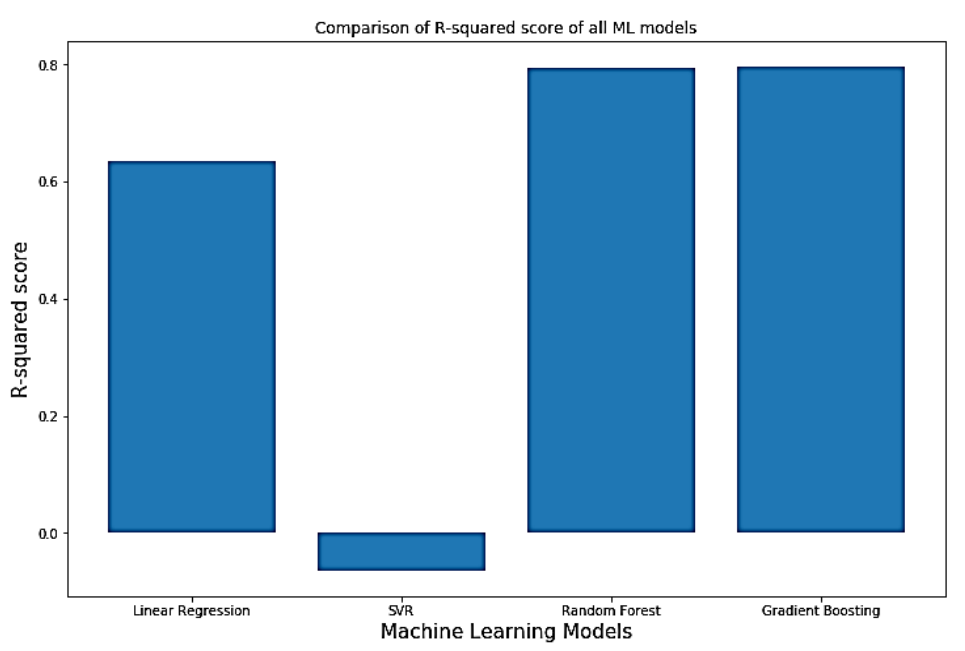

Figure 11 Results of AIC and Bic Before Log Transformation

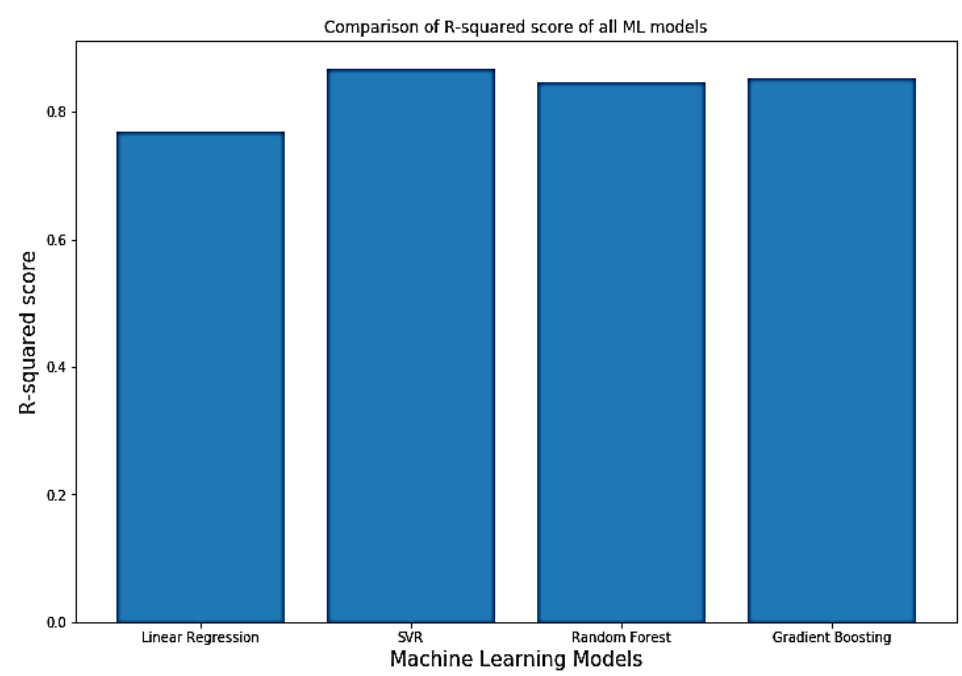

Figure 12 Results of PCA After Log Transformation

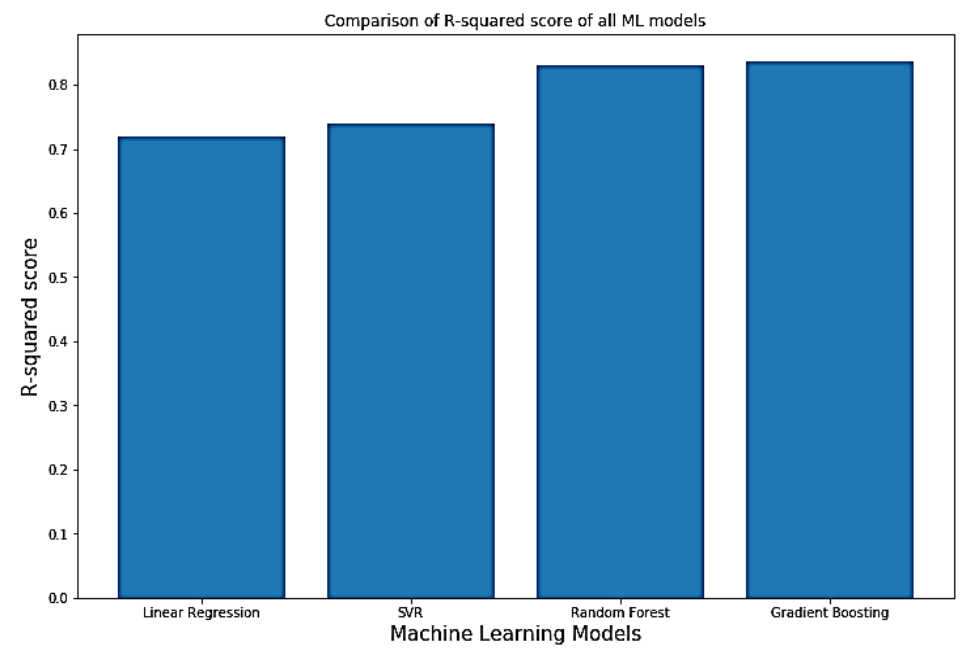

Figure 13 Results of AIC and BIC After Log Transformation 


\subsection{Winner Methodology}

Table 2 Results of Winner Algorithm Optimized by Ga

\begin{tabular}{|l|c|c|c|c|c|c|}
\hline \multicolumn{1}{|c|}{$\begin{array}{c}\text { Model } \\
\text { name }\end{array}$} & Gen & Pop & C & gamma & R-square & RMSE \\
\hline SVR & - & - & 1 & auto & $86 \%$ & .19 \\
\hline SVR & - & - & 10 & auto & $86 \%$ & .19 \\
\hline GA-SVR & 1 & 2 & 882.8 & .552 & $67 \%$ & .30 \\
\hline GA-SVR & 5 & 4 & 934.7 & .080 & & \\
\hline GA-SVR & 9 & 6 & 986.31 & .2169 & $74 \%$ & .26 \\
\hline
\end{tabular}

\section{CONCLUSION}

From the analysis that has been performed, it looks likely positive that the correlation between the three methodologies studied.

The experiment results show that before taking the log transformation of the dataset, both null hypotheses are not rejected. Applying AIC and BIC as feature selection techniques does not produce over 5\% lower error rates than using PCA in any algorithm. We do not reject null hypothesis 1, which is that using the feature selection techniques AIC and BIC will not allow the SVR algorithm to have an over 5\% lower RMSE than using the same algorithms with feature extraction in PCA. RMSE increased by $7.3 \%$ in RF and 6.3\% in GB, whereas liner regression and SVR are the highest.

In addition, we do not reject null hypothesis 2, which is that using the feature selection techniques AIC and BIC will not allow the algorithms to have an over 5\% higher R-squared score than using the same algorithms with feature extraction in PCA. The R-squared score in $\mathrm{RF}$ decreased by $4 \%$ and in GB, it decreased by $3 \%$, while the remaining algorithms increased more.

After applying log transformation to the dataset, the R-squared scores increased and the error reduced the model result overall. Still, the null hypothesis is not rejected, because the algorithms with AIC and BIC did not reduce RMSE and did not increase the which in comparison with the PCA result. With log transformation, SVR-PCA produced the highest Rsquared score and lowest RMSE.

In null hypotheses 1 , SVR increased by $28 \%$ in RMSE, while RF and GB increased by $4 \%$ and $4.5 \%$, respectively. With null hypotheses 2 , we did not reject it because all the algorithms have a lower R-squared score when using the AIC and BIC feature selection techniques than when using PCA by $17 \%, 1.9 \%$ and $1.7 \%$ with SVR, RF and GB, respectively.

This project compared the use of two experiments: first, AIC and BIC as feature selection techniques with four different machine learning algorithms, SVR, RF, GB and linear regression, and second, PCA as a feature extraction technique with the same algorithms. These experiments aimed to find the best housing price prediction model for the KC USA dataset. In the feature selection experiment, we chose seven features, where in the feature extraction experiment, we used 16 PCs as algorithm inputs. After the experiment, the results show that PCA had a better performance than feature selection with AIC and BIC. PCA with SVR and RBF Kernel achieved the highest R-squared scores at $86 \%$.

This project only uses AIC and BIC as feature selection methods. In the future, different selection methods can be used to conduct the same experiment. In addition, more work can be 
Managing and Mitigating Housing - Price Predictions using a Genetic Algorithm: A Case of King County in Washington, U.S.A

done to expand the number of features, such as feature aggregation, because the number of features is still small. Furthermore, the research suggests applying this method to housing prices.

\section{REFERENCES}

[1] Hess, T., Matt, C., Benlian, A., \& Wiesböck, F. (2016). Options for Formulating a Digital Transformation Strategy. MIS Quarterly Executive, 15(2), 123-139. Retrieved from https://www.researchgate.net/publication/291349362_Options_for_Formulating_a_Digital_Tr ansformation_Strategy

[2] W. Li, Y. Zhao, W. Meng, and S. Xu, "Study on the Risk Prediction of Real Estate Investment Whole Process Based on Support Vector Machine," in 2009 Second International Workshop on Knowledge Discovery and Data Mining, 2009, pp. 167-170.

[3] Y. El Hamzaoui and J. A. H. Perez, "Application of Artificial Neural Networks to Predict the Selling Price in the Real Estate Valuation Process," in 2011 10th Mexican International Conference on Artificial Intelligence, 2011, pp. 175-181.

[4] Yanli Zhao and Shuangshuang Jia, "The regression analysis and prediction of Real estate added value based on genetic algorithm," in MSIE 2011, 2011, pp. 944-946.

[5] H. Crosby, P. Davis, T. Damoulas, and S. A. Jarvis, "A spatio-temporal, Gaussian process regression, real-estate price predictor," in Proceedings of the 24th ACM SIGSPATIAL International Conference on Advances in Geographic Information Systems - GIS '16, 2016, pp. $1-4$.

[6] V. A. Salnikov and O. M. Mikheeva, "Models for Predicting Prices in the Moscow Residential Real Estate Market," Stud. Russ. Econ. Dev., vol. 29, no. 1, pp. 94-101, Jan. 2018.

[7] A. Varma, A. Sarma, S. Doshi, and R. Nair, "House Price Prediction Using Machine Learning and Neural Networks," in 2018 Second International Conference on Inventive Communication and Computational Technologies (ICICCT), 2018, pp. 1936-1939

[8] Hu Xiaolong and Zhong Ming, "Applied research on real estate price prediction by the neural network," in 2010 The 2nd Conference on Environmental Science and Information Application Technology, 2010, pp. 384-386.

[9] Wang, X., Wen, J., Zhang, Y. and Wang, Y., 2014. Real estate price forecasting based on SVM optimized by PSO. Optik-International Journal for Light and Electron Optics, 125(3), pp.14391443.

[10] Gu, J., Zhu, M. and Jiang, L., 2011. Housing price forecasting based on genetic algorithm and support vector machine. Expert Systems with Applications, 38(4), pp.3383-3386.

[11] Ethridge, D. E. (2004). Research Methodology in Applied Economics. Wiley.

[12] Njoroge, Ongeti, Kinuu, \& Kasomi. (2016). Does External Environment Influence Organizational Performance? The Case of Kenyan State Corporations. Management and Organizational Studies, 3(3). https://doi.org/10.5430/mos.v3n3p41.

[13] Saunders, M., Lewis, P., \& Thornhill, A. (2003). Research Methods for Business Students (3rd ed.) [Book]. Retrieved from https://www.worldcat.org/title/research-methods-for-businessstudents/oclc/50280078

[14] Frazer, L., \& Lawley, M. (2000). Questionnaire Design \& Administration: A Practical Guide. Retrieved from https://books.google.co.in/books?id=GnZgQgAACAAJ 